\title{
Diversidade e análise faunística de Sphingidae (Lepidoptera) em área de brejo e caatinga no Estado da Paraíba, Brasil
}

\author{
Maria Avany B. Gusmão \& Antonio José Creão-Duarte
}

Departamento de Sistemática e Ecologia, Universidade Federal da Paraíba. Campus I, 58059-900 João Pessoa, Paraíba, Brasil.E-mail: avanybezerra@uol.com.br; creao@dse.ufpb.br

\begin{abstract}
Diversity and faunistic analysis of Sphingidae (Lepdoptera) in areas of brejo and caatinga in Paraíba State, Brazil. Sphingidae assemblages of a highland humid forest (brejo) and a caatinga area were studied from specimens collected with light traps, during twelve months (March, 2000 to February, 2001), with the objective of knowing the diversity and establishing some parameters aiming at monitoring of these areas. Nineteen species were collected, fifteen from brejo and fourteen from caatinga. Ten species are common to both areas, five were collected only in brejo and four in caatinga. Callionima grisescens elegans (Gehlen, 1935) was the most abundant species in two areas. The distribution of species abundance in brejo follows the truncated log normal model. In caatinga the distribution pattern of abundance was not discriminated by the $\chi^{2}$ test, although indirect evidence indicates a tendency to the log series model. The assemblage structure (parameters) was estimated from the diversity indices. In brejo, where the eveness was larger, Shannon and Brillouin indices surpass those from caatinga. In this location, Simpson and Berger-Parker indices are much higher due to a larger dominance. The population fluctuation for both Sphingidae assemblages, shows that their populations are affected by rain distribution during the year, or more precisely, by the period of water replacement in the soil, as demonstrated by the water balance.
\end{abstract}

KEY WORDS. Diversity measures, light trap, water balance.

RESUMO. As comunidades de Sphingidae da área de brejo e área de caatinga foram estudadas com armadilhas luminosas, durante 12 meses (março/2000 a fevereiro/2001), com o objetivo de conhecer a diversidade e estabelecer alguns parâmetros para o monitoramento dessas áreas. Dezenove espécies foram coletadas, sendo 15 na área de brejo e 14 na área de caatinga. Dez espécies foram comuns às áreas, 15 foram coletadas apenas no brejo e quatro na caatinga. Callionima grisescens elegans (Gehlen, 1935) foi a espécie mais abundante nas duas áreas. A distribuição da abundância de espécies no brejo segue o modelo log normal truncado. Na caatinga o padrão de distribuição de abundância de espécies não foi discriminado pelo teste $\chi^{2}$ embora evidências indiretas indicam uma tendência para o modelo série log. Os parâmetros estruturais das assembléias foram estimados a partir de índices de diversidade. No brejo, enquanto a equitatividade foi maior, os índices de Shannon e Brillouin superam àqueles da caatinga. Nessa localidade, os índices de Simpson e Berger-Parker são muito maior devido a uma maior dominância. A flutuação populacional para ambas as assembléias, mostraram que suas populações são afetadas pela distribuição de chuvas durante o ano, ou mais precisamente, pelo período de reposição de água no solo, como demonstrado pelo balanço hídrico.

PALAVRAS CHAVE. Armadilha luminosa, balanço hídrico, medidas de diversidade.

A função de um programa de monitoramento é fornecer dados que possam ser utilizados no gerenciamento de reservas para restauração ou manutenção da composição, da estrutura e do funcionamento dos ecossistemas (FrankLIN et al. 1981 apud KREMEN 1992).

O papel desempenhado pelos invertebrados no meio ambiente fez com que os mesmos passassem a ser, cada vez mais, empregados como instrumentos auxiliares em estudos de meio ambiente, seja na conservação, no gerenciamento ambiental ou nas estratégias de monitoramento dos ecossistemas (BiLTon 1996, Drake 1996, Eyre 1996, Eyre \& Woodward 1996, Falk 1996, Fowles 1996).

As análises de fauna têm sido empregadas com propósitos diversos. No meio agronômico, em geral, objetivam a elaboração de programas integrados de controle de pragas (LARA et al. 1977, Silveira Neto et al. 1977, Pereira 1994, Pereira et al.

Revista Brasileira de Zoologia 21 (3): 491-498, setembro 2004 
1995). Em estudos ecológicos, têm por finalidade a caracterização e a estrutura de comunidades, assim como a aval iação de impactos a partir da comparação de dados decomposição e abundância defauna, obtidos em períodos diversos ( LAROcA et al. 1989, Hluchy 1990, Andersen 1990, Lubeck et al. 1995, SilveirA Neto et al. 1995, Archer 1996, Camargo 1999, Marinoni \& Dutra 1991, 1996, MARINONI et al. 1997, 1999).

A expansão dasatividades humanas, sobretudo nasúltimas décadas, tem exercido forte pressão sobre as áreas de reserva natural, com grave redução da diversidade biológica. Parece que esta constatação vem corroborar a afirmação deM AY (1988) de que a conservação biológica éuma ciência com tempo limitado.

As pressões antrópicas exercidas sobre as áreas de matas de brejo ecaatinga, ten dem a se intensificar com a diminuição espacial da vegetação, com graves conseqüências sobre a fauna. O propósito deste trabalho foi conhecer a diversidade de Sphingidae destas árease, ao mesmo tempo, disponibilizar informações demedidas defauna que possam ser utilizadascomo dados históricos deste grupo taxonômico, para as áreas estudadas, em trabal hos futuros de conservação.

\section{MATERIAL E MÉTODOS}

O trabalho foi desenvolvido em duas áreas do Estado da Paraíba: Mata de Pau Ferro (brejo) e Mata de Paus Brancos (caatinga). A primeira éuma Reserva Ecológica Estadual com 607 ha, típica floresta de brejo, que se assemelha aos remanescentes da floresta verde da região costeira da Paraíba. Esten de-se entre 400 a $600 \mathrm{~m}$ de altitude, com precipitação média de $1.400 \mathrm{~mm}$. Local iza-se $5 \mathrm{~km}$ a oeste da cidade de Areia, Paraíba, e faz parte da bacia hidrográfica do reservatório deágua Vaca Brava. As médias anuais de temperatura e umidade rel ativa do ar são, respectivamente, $22^{\circ} \mathrm{C}$ e $85 \%$. A segunda área apresenta características típicas de caatinga arbustiva, tem 1.190 ha, com cerca de 10\% de vegetação nativa. Localiza-se na microbacia do riacho São Pedro, Distrito de Catolé da Boa Viagem, M unicípio de Campina Grande, Paraíba. A precipitação anual pode variar de 400 a $800 \mathrm{~mm}$. A média anual de temperatura varia de 25 a 28 ㅇ C e a umidade relativa é de $50 \%$.

As col etas ocorreram no período de março de 2000 a fevereiro de 2001, a cada novilúnio. Cada coleta compreendia dois períodos de 12 horas, sempre das 18:00 até as 6:00 h do dia seguinte. O primeiro período iniciava-se no dia que antecedia a lua nova e o segundo, no dia da lua nova.

Em cada uma das áreas de estudo foram utilizadas duas armadi Ihas luminosas (Luiz deQuei roz com modificações), equipadas com luz negrafluorescente (UV) de $20 \mathrm{~W}$, al imentadas por baterias de $45 \mathrm{~A}$ e instaladas a $1,5 \mathrm{~m}$ do solo nas coordenadas: 0721'26"S e 3603'33"W; 0721'27"S e 36003'10"W (caatinga); 0658'34"Se 3545'10"W; 0658'23" e 3544'54"W (brejo).

O material coletado encontra-se depositado na Coleção Entomológi ca do Departamento de Sistemática e Ecologia da Universidade Federal da Paraíba.
Os índices de Shannon, Brillouin, Berger-Parker e Simpson foram utilizados para avaliar a diversidadeea eqüitatividade. Exceto para Brillouin, calculado mediante uso do M ETLab (pacote estatístico) todos os demais índices foram obtidos através do pacote estatístico SPSS 9,0/1996, também utilizado nos cálculos de correlação linear (método de Pearson) entre a flutuação populacional dos Sphingidae e os fatores meteorológicos, inclusive os de deficiência hídrica do solo.

Os padrões de distribuição de abundância de espécies, observados nas áreas de estudo (Fig. 1), foram testados junto aos modelos série log e log normal (truncado).

Osdadosmeteorológicosforam obtidosjunto ao Laboratório de Recursos Hídricose Sensoriamento Remoto da Paraíba (LM RH). O bal anço hídrico foi calculado segundo o método de TORTOWHATER \& MATHER (1955), com retenção hídrica estimada em $100 \mathrm{~mm}$.

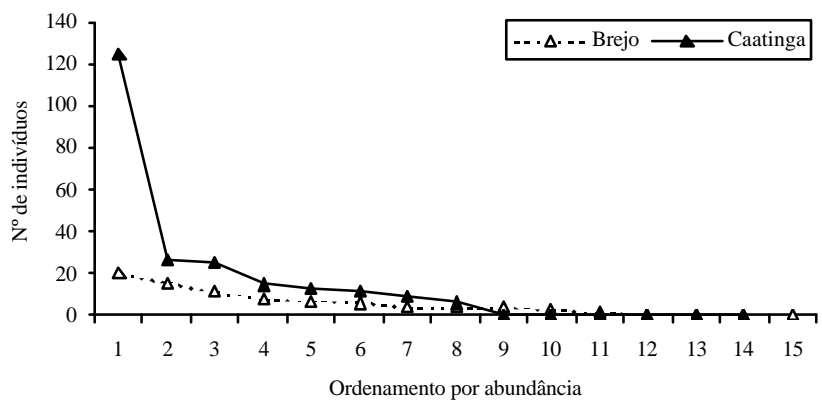

Figura 1. "Rank" de abundância das espécies de Sphingidae coletadas em área de Caatinga e Brejo, com armadilha luminosa, nos novilúnios de março/2000 a fevereiro/2001.

\section{RESULTADOS E DISCUSSÃO}

Nas duas áreas de estudo foram registradas 19 espécies de Sphingidae de 13 gêneros, acomodados em seis tribos, totalizando 326 indivíduos. Dez espécies são comuns às duas áreas e nove foram exclusivas, sendo cinco para o brejo equatro para a caatinga (Tab. I).

Apesar da padronização do uso da armadil ha luminosa nas áreas estudadas, o raio da ação da luz ul travioleta, provavelmente, foi influenciado pela diferença fisionômica entre as áreas estudadas. Este fator deve ser considerado, pois interfere no taman ho das amostras (LAROCA et al. 1989, M ARINONI \& D UTRA 1996). Isto talvez explique a disparidade entre o número de indivíduos col etados no brejo (89) e na caatinga (237). Maior diversidadeem ambientes florestados com menor abundância em relação às formações abertas foi também observado por Duellman (1990) e Zimmerman \& Rodrigues (1990).

A composição da assembléia de Sphingidae dasáreas de brejo e caatinga (Tab. II), apresentou maior proporção de espé cies e indivíduos para as tribos Dilophonotini eSphingini, res- 
Tabela I. Ocorrência de espécies de Sphingidae (S) e número de indivíduos (N) por área de estudo, Caatinga e Brejo, Paraíba, Brasil, coletados com armadilha luminosa nos novilúnios de março/2000 a fevereiro/2001.

\begin{tabular}{|c|c|c|c|c|}
\hline \multirow{2}{*}{ Espécies } & \multicolumn{2}{|c|}{ Caatinga } & \multicolumn{2}{|c|}{ Brejo } \\
\hline & $\mathrm{S}$ & $\mathrm{N}$ & $\mathrm{S}$ & $\mathrm{N}$ \\
\hline \multicolumn{5}{|l|}{ Sphinginae } \\
\hline \multicolumn{5}{|l|}{ Sphingini } \\
\hline Manduca rustica rustica (Fabricius, 1775) & $\mathrm{x}$ & 1 & $\mathrm{x}$ & 1 \\
\hline Manduca sexta paphus (Cramer, 1779) & $\mathrm{x}$ & 13 & $x$ & 16 \\
\hline Neogene dynaeus (Húbner, [1827]-[1831]) & $\mathrm{X}$ & 27 & $\mathrm{x}$ & 4 \\
\hline \multicolumn{5}{|l|}{ Acherontini } \\
\hline Agrius cingulata (Fabricius, 1775) & $\mathrm{x}$ & 11 & $\mathrm{x}$ & 11 \\
\hline \multicolumn{5}{|l|}{ Smerintinae } \\
\hline \multicolumn{5}{|l|}{ Ambulycini } \\
\hline Protambulyx strigilis (Linnaeus, 1771) Macroglossinae & $\mathrm{X}$ & 1 & $\mathrm{x}$ & 8 \\
\hline \multicolumn{5}{|l|}{ Dilophonotini } \\
\hline Callionima grisescens elegans (Gehlen, 1935) & $\mathrm{x}$ & 125 & $\mathrm{x}$ & 20 \\
\hline Callionima parce (Fabricius, 1775) & - & - & $x$ & 6 \\
\hline Enyo lugubris lugubris (Linnaeus, 1771) & $x$ & 6 & - & - \\
\hline Erinnyis ello ello (Linnaeus, 1758) & $x$ & 15 & $\mathrm{x}$ & 7 \\
\hline Erinnyis allope allope (Drury, 1773) & $x$ & 1 & - & - \\
\hline Isognathus menechus (Boisduval, 1875) & $x$ & 1 & $x$ & 1 \\
\hline Perigonia lusca lusca (Fabricius, 1777) & - & - & $x$ & 1 \\
\hline Perigonia pallida Rotschild \& Jordan, 1903 & $\mathrm{x}$ & 1 & - & - \\
\hline Pseudosphinx tetrio (Linnaeus, 1771) & - & - & $x$ & 3 \\
\hline \multicolumn{5}{|l|}{ Philampelini } \\
\hline Eumorpha labruscae (Linnaeus, 1758) & - & - & $x$ & 1 \\
\hline Eumorpha vitis vitis (Linnaeus, 1758) & $x$ & 9 & $x$ & 4 \\
\hline \multicolumn{5}{|l|}{ Macroglossini } \\
\hline Hyles euphorbiarum (Guérin-Méneville \& Percheron, 1835) & $\mathrm{X}$ & 25 & $x$ & 4 \\
\hline Xylophanes pluto (Fabricius, 1777) & $x$ & 1 & - & - \\
\hline Xylophanes tersa tersa (Linnaeus, 1771) & - & - & $\mathrm{x}$ & 2 \\
\hline Total & 14 & 237 & 15 & 89 \\
\hline
\end{tabular}

Tabela II. Espécies (S) e indivíduos (N) de Sphingidae por tribo, em valores absolutos (Ab) e percentuais (\%), coletadas na caatinga e brejo, com armadilha luminosa, nos novilúnios de março/2000 a fevereiro/2001.

\begin{tabular}{|c|c|c|c|c|c|c|c|c|c|c|c|c|}
\hline \multirow{3}{*}{ Tribos } & \multicolumn{6}{|c|}{ Espécies } & \multicolumn{6}{|c|}{ Indivíduos } \\
\hline & \multicolumn{2}{|c|}{ Caatinga } & \multicolumn{2}{|c|}{ Brejo } & \multicolumn{2}{|c|}{ Total } & \multicolumn{2}{|c|}{ Caatinga } & \multicolumn{2}{|c|}{ Brejo } & \multicolumn{2}{|c|}{ Total } \\
\hline & $A b$ & $\%$ & $A b$ & $\%$ & $A b$ & $\%$ & $A b$ & $\%$ & $\mathrm{Ab}$ & $\%$ & $A b$ & $\%$ \\
\hline Acherontinini & 1 & 7,14 & 1 & 6,67 & 1 & 5,26 & 11 & 4,64 & 11 & 12,36 & 22 & 6,75 \\
\hline Ambulycini & 1 & 7,14 & 1 & 6,67 & 1 & 5,26 & 1 & 0,42 & 8 & 8,99 & 9 & 2,76 \\
\hline Dilophonotini & 6 & 42,86 & 6 & 40,00 & 9 & 47,37 & 149 & 62,87 & 38 & 42,70 & 187 & 57,36 \\
\hline Macroglossini & 2 & 14,29 & 2 & 13,33 & 3 & 15,79 & 26 & 10,97 & 6 & 6,74 & 32 & 9,82 \\
\hline Philampelini & 1 & 7,14 & 2 & 13,33 & 2 & 10,59 & 9 & 3,80 & 5 & 5,62 & 14 & 4,29 \\
\hline Sphingini & 3 & 21,43 & 3 & 20,00 & 3 & 15,79 & 41 & 17,30 & 21 & 23,60 & 62 & 19,02 \\
\hline Total & 14 & 100 & 15 & 100 & 19 & 100 & 237 & 100 & 89 & 100 & 326 & 100 \\
\hline
\end{tabular}


pectivamente. As proporções verificadas assemelham-seàsobtidas para outros biótopos tropicais (LARocA \& MieLKE 1975, Laroca et al. 1989, Corral \& Sierra 1994, Motta et al. 1998).

O Teste $\chi^{2}$ indicou que os dados observados para a área de brejo concordam com os esperados para o modelo sérielog normal. Para os dados observados na área de caatinga o teste $\chi^{2}$ não discriminou que modelo os dados seguem (Tab. III). Conjunto de dados pequenos pode apresentar este comportamento ou como Magurran (1988) e M artin Piera (1997) destacaram, serem discriminados por ambos os modelos.

Se o modelo de distribuição de abundância de espécies log normal truncado é exibido por comunidades em al to grau de equilíbrio (M Inshall et al. 1985, Kevan et al. 1997), o modelo série log é visto como um indicador de ambientes alterados, onde poucos fatores determinam maior dominância e menor eqüitatividade entre as espécies (M AGURRAn 1988). Esses indicadores do model o série log normal são observados na caatinga, onde ocorre grande dominância deCallionimagrisescens el egans (Gehlen, 1935) (Tab. I) e a área passa por grande processo de descaracterização para assentamentos agrícolas.

Índices de diversidade

Os índices de diversidade e uniformidade são apresentados na tabela IV. Para o brejo, cujo padrão de distribuição de abundância segueo modelo log normal (truncada), a proporcionalidade de abundância émel hor definidae, portanto, os índices de diversidade de Shannon e Brillouin apresentam valores maiores, quando comparados com os obtidos para a área de caatinga. Sempre que se compara valores obtidos por esses dois índices, para um mesmo conjunto de dados, observa-se que os resultados para Brillouin são sempre menores que os obtidos para Shannon, como se pode observar com os Ctenuchidae de M ARINONI \& DUTRA (1996). Isto decorre de Brillouin descrever uma coleção conhecida, enquanto Shannon estima a diversidade a partir da porção amostrada e não amostrada da comunidade
(MagurRan 1988, Stiling 1999).

Para a área de caatinga, cujo padrão de distribuição de abundância sugere uma tendência para o modelo série log, a dominância está caracterizada e, portanto, osíndices de diversidade de Simpson e Berger-Parker têm valores maiores.

\section{Balanço hídrico}

O bal anço hídrico contabiliza as entradas esaídas deágua no solo, o quetorna possível estimar aágua contida no mesmo a cada período do ano. Tem gran de aplicação no meio agronômico, sobretudo, para orientar os intervalos de irrigação, planejamento dos recursos deágua, classificação climática eem estudos do meio ambiente (M отA 1977, Xu 1999, Evans et al. 1999). Como os Sphingidae desenvolvem a fase pupa no solo, provavel mente os teores de umidadeno solo determinam a passagem para a fase adulta. (Janzen 1983, Meerman 1999, Kitching \& Cadiou 2000).

Observando o gráfico da flutuação populacional dos Sphingidae na caatinga (Fig. 2), a ausência de espécies para os períodos mais críticos da seca (setembro-dezembro), não representa na realidade ausência local da espécie, mas ausência do adulto. Possivelmenteestes insetos permanecem sob o solo,

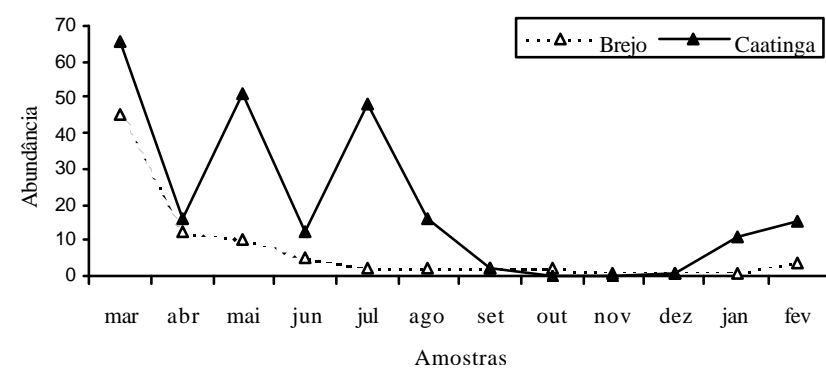

Figura 2. Flutuação populacional de Sphingidae em área de Caatinga e Brejo, Paraíba, Brasil. Dados observados no período de março/2000 a fevereiro/2001, utilizando armadilha luminosa.

Tabela III. Teste de aderência com valores observados e esperados, série log e log normal (truncada), entre classes de abundância de espécies de Sphingidae coletados com armadilha luminosa, na Caatinga (1) e Brejo (2), Paraíba, Brasil, nos novilúnios de março/2000 a fevereiro/2001.

\begin{tabular}{|c|c|c|c|c|c|c|}
\hline \multirow{2}{*}{ Área de Estudo } & \multicolumn{3}{|c|}{ Série Log } & \multicolumn{3}{|c|}{ Série normal truncada } \\
\hline & Observado & Esperado & $\chi^{2}$ & Observado & Esperado & $\chi^{2}$ \\
\hline 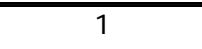 & 14 & 14 & $6,6,41$ & 14 & 19 & 7,18 \\
\hline 2 & 15 & 15 & 1,89 & 15 & 15,6 & 0,18 \\
\hline
\end{tabular}

Sendo: $X=0,98645349 ;(=3,255$ - Série log; $(=20,16$ - Série Normal, para a Caatinga. $X=0,94512219 ;(=5,168$ - Série log; $(=33,28$

- Série log normal (truncada), para o Brejo.

Tabela IV. Índices de diversidade e uniformidade de Sphingidae em ecossistema de Caatinga (1) e Brejo (2), Paraíba, Brasil. (S) número de espécies, (N) número de indivíduos, (HB) Índice de diversidade de Brillouin, (H') Índice de diversidade de Shannon; (HBE) Índice de uniformidade de Brillouin, (H'E) Índice de uniformidade Shannon, (BP) índice de Berger \& Parker, (UBP) Índice de uniformidade de Berger \& Parker, (D) Índice de Simpson, (1/D) Índice de uniformidade de Simpson.

\begin{tabular}{ccccccccccc}
\hline Área & $\mathrm{S}$ & $\mathrm{N}$ & $\mathrm{HB}$ & $\mathrm{HBE}$ & $\mathrm{H}^{\prime}$ & $\mathrm{H}$ 'E & BP & UBP & D & $1 / \mathrm{D}$ \\
\hline 1 & 14 & 237 & 1,56 & 0,62 & 1,65 & 0,63 & 0,527 & 1,896 & 0,310 & 3,218 \\
2 & 15 & 089 & 2,08 & 0,86 & 2,32 & 0,86 & 0,225 & 4,450 & 0,115 & 8,683 \\
\hline
\end{tabular}


na fase pupal, até que as condições de umidade sejam favoráveis à emergência dos adultos. Muitas das estratégias de sobrevivência das espécies na caatinga estão ligadas aos teores de umidade do solo.

Apesar do período de seca iniciar-se em agosto e estender-se até dezembro, excepcionalmente, no ano 2001, choveu $308,1 \mathrm{~mm}$ em agosto e 219,9 mm em setembro na área de brejo (Tab. V). Muito embora haja coincidência nos dois meses de menor índicede precipitação pluviométrica (outubro enovembro) entre as duas áreas, os teores de água armazenada no solo diferem substancialmente entre elas. Na área de brejo o total de precipitação para o período (março/2000 a fevereiro/2001) foi de 1.866,6mm; houve excedente hídrico durante seis meses e deficiência hídrica durante cinco meses (Tab. V). Na área de caatinga o total de precipitação para o mesmo período foi de $582,6 \mathrm{~mm}$, mas não houve excedente hídrico e a deficiência se estendeu por dez meses, sendo que em nenhum mês do ano houve água armazenada no solo para capacidade de reten ção hídrica estipulada (Tab. VI).

Nas duasáreas a flutuação populacional al cança osmaiores picos logo após a época de deficiência hídrica, quando o solo recebeágua de reposição e, com isso, aumenta seu teor de umidade. De certa forma é intrigante o fato de ter sido capturado maior número de indivíduos, na época mais úmida, na

Tabela V. Balanço hídrico para área de Brejo, Areia, Paraíba, Brasil. Dados de março/2000 a fevereiro/2001. (EP) Evapotranspiração potencial, (P) pluviosidade, (Neg. Acum.) negativa acumulada, (Arm.) armazenamento, (Alt.) alteração, (ER) evapotranspiração real, (Def.) deficiência, (Exc.) excedente.

\begin{tabular}{|c|c|c|c|c|c|c|c|c|c|c|c|c|}
\hline Meses & Temp. & Nomograma & Correção & $\mathrm{EP} \mathrm{mm}$ & $\mathrm{P} \mathrm{mm}$ & $P$ - EP mm & Neg. Acum. & Arm. mm & Alt. $\mathrm{mm}$ & ER $\mathrm{mm}$ & Def. mm & Exc. $\mathrm{mm}$ \\
\hline Mar & 24,80 & 108 & 1,04 & 112,32 & 119,2 & 6,88 & 220,00 & 10 & 7 & 112,30 & 0 & 0 \\
\hline Abr & 24,15 & 102 & 1,00 & 102,00 & 269,4 & 167,40 & 0 & 100 & 90 & 102,00 & 0 & 74,40 \\
\hline Mai & 22,70 & 85 & 1,02 & 86,70 & 120,5 & 33,80 & 0 & 100 & 0 & 86,70 & 0 & 33,80 \\
\hline Jun & 22,60 & 84 & 0,99 & 83,16 & 323,5 & 240,34 & 0 & 100 & 0 & 83,16 & 0 & 240,34 \\
\hline Jul & 21,90 & 86 & 1,02 & 87,72 & 349,7 & 261,98 & 0 & 100 & 0 & 87,72 & 0 & 261,98 \\
\hline Ago & 21,50 & 84 & 1,03 & 86,52 & 308,1 & 221,58 & 0 & 100 & 0 & 86,52 & 0 & 221,58 \\
\hline Set & 22,10 & 81 & 1,00 & 81,00 & 219,9 & 138,90 & 0 & 100 & 0 & 81,00 & 0 & 138,90 \\
\hline Out & 23,50 & 82 & 1,05 & 86,10 & 19,9 & $-66,20$ & 66,20 & 51 & -49 & 68,90 & 17,20 & 0 \\
\hline Nov & 24,50 & 104 & 1,03 & 107,12 & 25,0 & $-82,12$ & 148,32 & 22 & -29 & 54,00 & 53,12 & 0 \\
\hline Dez & 24,80 & 108 & 1,06 & 114,48 & 45,1 & $-69,38$ & 217,70 & 11 & -11 & 56,10 & 58,38 & 0 \\
\hline Jan & 24,50 & 104 & 1,06 & 110,24 & 46,6 & $-63,64$ & 281,34 & 6 & -5 & 51,60 & 58,64 & 0 \\
\hline Fev & 26,60 & 126 & 0,95 & 119,70 & 19,7 & $-100,00$ & 381,34 & 3 & -3 & 22,70 & 97,00 & 0 \\
\hline Anual & 23,64 & & & 1177,06 & 1866,6 & 689,54 & & & 0 & 892,70 & 284,34 & \\
\hline
\end{tabular}

Tabela VI. Balanço hídrico para área de Caatinga, Catolé Boa Vista, Campina Grande, Paraíba, Brasil. Dados de março/2000 a fevereiro/2001. (EP) Evapotranspiração potencial, (P) pluviosidade, (Neg. Acum.) negativa acumulada, (Arm.) armazenamento, (Alt.) alteração, (ER) evapotranspiração real, (Def.) deficiência, (Exc.) excedente.

\begin{tabular}{ccccrrrrrrrrrr}
\hline \hline Meses & Temp. & Nomograma & Correção & E $\mathrm{mm}$ & \multicolumn{1}{c}{ P mm } & P - EP mm & Neg. Acum. Arm. mm & Alt. mm & ER mm & Def. mm & Exc. mm \\
\hline \hline Mar & 26,79 & 135 & 1,04 & 140,40 & 143,9 & 3,50 & 253,00 & 6,5 & 3,5 & 140,4 & 0 & 0 \\
Abr & 25,58 & 128 & 1,00 & 128,00 & 170,6 & 42,60 & 69,00 & 49,1 & 42,6 & 128,0 & 0 & 0 \\
Mai & 25,14 & 112 & 1,02 & 114,24 & 48,0 & $-66,24$ & 69,00 & 25,0 & $-24,1$ & 72,1 & 42,14 & 0 \\
Jun & 24,23 & 94 & 0,99 & 93,06 & 33,9 & $-59,16$ & 135,24 & 14,0 & $-11,0$ & 44,9 & 48,16 & 0 \\
Jul & 23,65 & 90 & 1,02 & 91,80 & 51,0 & $-40,80$ & 194,40 & 9,0 & $-5,0$ & 56,0 & 35,80 & 0 \\
Ago & 24,09 & 91 & 1,03 & 93,73 & 39,4 & $-54,33$ & 235,20 & 5,0 & $-4,0$ & 43,4 & 50,33 & 0 \\
Set & 24,60 & 103 & 1,00 & 103,00 & 21,0 & $-82,00$ & 289,53 & 3,0 & $-2,0$ & 23,0 & 80,00 & 0 \\
Out & 26,30 & 128 & 1,05 & 134,40 & 4,1 & $-130,30$ & 371,53 & 3,0 & 0 & 4,1 & 130,30 & 0 \\
Nov & 26,90 & 129 & 1,03 & 132,87 & 1,8 & $-131,07$ & 501,83 & 3,0 & 0 & 1,8 & 131,07 & 0 \\
Dez & 26,76 & 128 & 1,06 & 135,68 & 44,9 & $-90,78$ & 632,90 & 3,0 & 0 & 44,9 & 90,78 & 0 \\
Jan & 26,24 & 125 & 1,06 & 132,50 & 23,6 & $-108,90$ & 723,68 & 3,0 & 0 & 23,6 & 108,90 & 0 \\
Fev & 27,71 & 144 & 0,95 & 136,80 & 0,4 & $-136,40$ & 832,58 & 3,0 & 0 & 0,4 & 136,40 & 0 \\
\hline \hline Anual & 25,66 & & & 1436,50 & 582,6 & $-853,88$ & 968,98 & & 0 & 582,6 & 853,88 & 0 \\
\hline
\end{tabular}


caatinga do que no brejo. A dominância de Callionima grisescecens el egans na caatinga éa responsável por esta diferença, o quedemonstra uma partilha derecurso menos equilibrada nesta área que no brejo. Uma outra explicação pode decorrer do método de amostragem, sendo a caatinga um ambiente mais aberto, com relação ao brejo, favorece mais a dispersão da luz que, assim, agiria de forma mais eficiente na atração dos insetos (M ARINONI \& DUTRA 1991, 1996)

\section{Sazonalidade}

As áreas em estudo apresentam duas estações climáticas bem definidas: uma seca e outra úmida marcada pela ocorrência das chuvas. Na estação seca, a paisagem da caatinga muda substancialmente, as plantas perdem as fol has e a vegetação adquire uma tonalidade esbranquiçada, daí o nome do lugar, Mata de Paus Brancos.

A flutuação dos Sphingi dae na área de caatinga mostra que a população ocorre em maior número no período quevai de março a julho (Fig. 2). Os dois pontos de baixa ocorrência (abril ejunho) não são indicadores reais da flutuação populacional das espécies, pois decorrem de fenômeno ligado ao tempo (chuva), no dia de coleta. Na época seca os Sphingidae estiveram ausentes nas coletas de outubro e novembro, sendo coletado apenas um indivíduo em dezembro. No brejo há um pico em março e os meses de mais baixa freqüência foram de novembro a janeiro (Fig. 2). Esta relação dos picos populacionais com o período chuvoso foi, também, observada para os Sphingidae da Serra do M ar ( Laroca et al. 1989), da Costa Rica (Haber \& Frankie 1989) e Belize (M Eerman 1999).

Na caatinga, a flutuação população dos Sphingidaemostrou correlação positiva significativa ao nível de $0,01 \mathrm{com}$ a pluviosidade $(r=0,709)$ e correlação negativa moderada com a temperatura máxima ( $r=-0,70 ; p=0,123$ ) (Fig. 3). No brejo, a flutuação populacional dos Sphingidaenão apresentou corre lação com nenhum fator meteorológico. Temperaturas máximas e pluviosidade são as variáveis meteorológicas mais correlacionadas com as el evações populacionais de Sphingidae (Grant 1983, Stradling et al. 1983, Ferreira et al. 1986, M arinoni et al. 1999).

A acentuada queda do número de in divíduos coletados nos meses de maio a dezembro e outubro a fevereiro, para a caatinga e o brejo, respectivamente, pode estar associada à de ficiência hídrica do solo. A abundância verificada nos referidos meses apresenta correlação negativa significativa com a deficiência hídrica do solo (Fig. 4), sendo ( $r=-0,623 ; p=0,031)$ para o brejo com significância de 0,05 e $(r=-0,743 ; p=0,006)$ para a caatinga com significância de 0,01.

\section{AGRA DECIMENTOS}

Os autores agradecem à Profa MSc. Catarina M otta, pela val iosa ajuda na identificação das espécies. Ao Prof. Dr. Renato Marinoni, pelas críticas e sugestões. A CAPES pela concessão de bolsa à primeira autora.

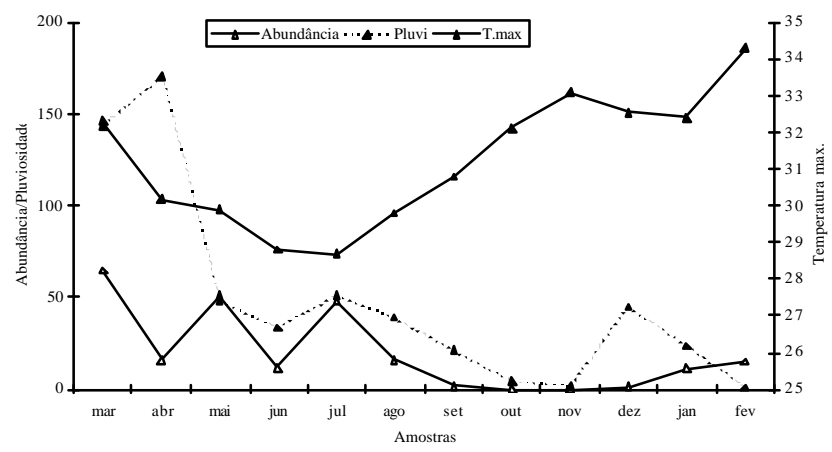

Figura 3. Correlação de Pearson positiva significativa a 0,01 da flutuação populacional de Sphingidae com a pluviosidade e correlação negativa moderada com temperatura máxima em área de Caatinga, Paraíba, Brasil. Dados observados no período de março/2000 a fevereiro/2001, utilizando armadilha luminosa.

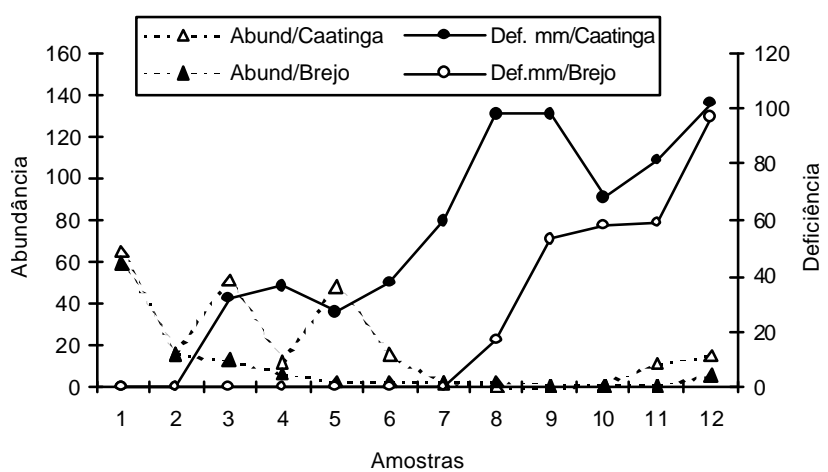

Figura 4. Correlação negativa significativa da deficiência hídrica do solo com a abundância mensal de Sphingidae em área de $\mathrm{Ca}$ atinga e Brejo, Paraíba, Brasil. Dados observados no período de março/2000 a fevereiro/2001.

\section{REFERÊN CIAS BIBLIOGRÁ FICAS}

Andersen, A.N. 1990. The use of ant communities to evaluate change in australian terrestrial ecosystems: a review and a recipe. Ecological Soci ety of Australia, Villavagen, 16: 34757.

Archer, M.E. 1996. The use of solitary wasps and bees in site assessment for wildlife conservation, p. 14-17. In: M.D. EYRE (Ed). Environmental monitoring, surveilance and conservation using invertebrates. Benton, EMS Publications, VIII+101p.

BıtTon, D.T. 1996. Myriapods, I sopods and Molluscs - Useful for Enviroment Assessment?, p. 18-21. In: M.D. EYRE (Ed). Enviromental Monitoring, Surveilance andConservation U sing I nvertebrates. Benton, EMSPublications, VIII+101p.

CAMARGo, A.J.A. 1999. Estudo comparativo sobre a composição ea diversidade delepidópterosnoturnos em cinco áreas da 
Região dos Cerrados. RevistaBrasileira Zoologia, Curitiba, 16 (2): 369-380.

Corral, J.R.A. \& J.R.A. Sierra. 1994. Lista de los Sphingidae (Lepidoptetra) de el valle, Merida, Venezuela. Boletín de Entomología Venezolana, N.S., Maracay, 9 (2): 139-149.

DraKe, C.M. 1966. English Nature's approach to monitoring invertebrates, p. 67-71. In: M.D. EYRE (Ed). Environmental monitoring, surveilance and conservation using invertebrates. Benton, EMSPublications, VIII+101p.

Duellman, W.E. 1990. Herpetofaunas in Neotropical Rainforests: comparativecomposition, history, and resource use, p. 455505. In: A.H. Gentry (Ed.). Four Neotropical Rainforest. New Haven, Yale University Press, 640p.

EYRE, M.D. 1996. Environmental monitoring, surveilance and conservation using invertebrates. Benton, EMS Publications, 101p.

Eyre, M.D. \& J.W. Woodward. 1996. Spiders in environmental surveillance and site assessment, p. 26-28. In: M.D. EYRE (Ed). Environmental monitoring, surveilancean d conservation usinginvertebrates. Benton, EMSPublications, VIII+ 101p.

Evans, S.P.; T.R. MAYR; J.M. H ollis \& C.D. Brown . 1999. SWBCM: a soil water balance capacity model for environmental applicationsin theUK. Ecological M odelling, Copenhagen, 121: 17-49.

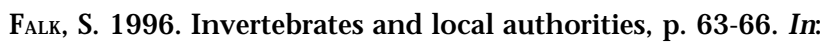
M.D. EYRE (Ed). Environmental monitoring, surveilance and conservation using invertebrates. Benton, EMS Publications, VIII+101p.

FerReira, P.S.F; D. dos S. Martins \& N. Hübner. 1986. Levantamento, flutuação e análise entomofaunística em mata remanescente da Zona da Mata, Viçosa, Minas Gerais. I. Sphingidae: Lepidoptera. Revista Ceres, Viçosa, 33 (190): 516-527.

FowLES, A.P. 1996. Experiences of invertebrate monitoring in wales, p. 77-79. In: M.D. EYRE (Ed). Environmental monitoring, surveilance and conservation usinginvertebrates. Benton, EMSPublications, VIII+101p.

Grant, V. 1983. The systematic and geographical distribution of hawkmoth flowers in thetemperate north american flora. Botical Gazeta, Chicago, 144 (3): 439-449.

Haber, W.A. \& G.W. Frankie. 1989. A tropical hawkmoth community: Costa Rican dry forest Sphingidae Biotropica, North Eagleville Road, 21 (2): 155-172.

HLuchy, M. 1990. Changes in the compositionsand abundance of selected families of Lepidoptera inhabiting the Pavlovské vrchy Hills during the $20^{\text {th }}$ century. Acta Entomologica Bohemosl ovaciae, Praha, 87:278-289.

JANZEN, D.H. 1983. Insects, p. 619-645. In: D.H. JANZEN (Ed). Costa Rica Natural History. Chicago, University of Chicago Press, $\mathrm{XI+816p.}$

KeVAn, P.G.; C.F. Greco \& S. Belaoussoff. 1997. Log-normality of biodiversity and abundance in diagnosis and measuring of ecosystemic health: pesticide stress on pollinators on blueberry heaths. Journal of Applied Ecology, Oxford, 34: 11221136.

KITCHING, I.J. \& J.-M. CADIOU. 2000. Hawkmoths of the world. An annotated and illustrated revisionary checklist (Lepidoptera: Sphingidae). London, Cornell University Press, VIII+227p.

KREMEN, C. 1992. Assessing the indicator properties of species assemblages for natural areas monitoring. Ecological Applications, Washington, 2 (2): 203-217.

Lara, F.M.; S.A. Bortoli \& E.A. Oliveira. 1977. Flutuações populacionais de alguns insetos associados ao Citrus sp e suas correl ações com fatores meteorológicos. Científica, São Paulo, 5 (2): 134-143.

Laroca, S. \& O.H.H. Mielke. 1975. Ensaios sobre ecologia de comunidade em Sphingidae na Serra do Mar, Paraná, Brasil (Lepidoptera). Revista Brasileira deBiologia, Curitiba, 35 (1): 1-18.

Laroca, S.; V.O. Becker \& F.C.V.ZAnella. 1989. Diversidade, abundância rel ativa efenologia em Sphingidae (Lepidoptera) na Serra do Mar (Quatro Barras, PR), sul do Brasil. Acta Biológica Paranaen se, Curitiba, 18 (1-4): 13-53 [1990].

LübeCK, G.M.; J.V. Olivera \& R.P. DeAlmeida. 1995. Análisefaunística de lepidópteros col etados em duas comunidades agrícoIas na Zona da M ata norte de Pernambuco. Anais da Sociedade Entomológica do Brasil, Londrina, 24 (2): 353-370.

MagurRan, A.E. 1988. Ecological Diversity and Its Measurement. Princeton, Princeton University Press, $X+179 p$.

MARINoni. R.C. \& R.R.C. D utRA. 1991. Levantamento da fauna entomológica no Estado do Paraná. I. Introdução. Situações climática e florística de oito pontos de coleta. Dados faunísticos de agosto de 1986 a julho de 1987. Revista Brasil eira Zool ogia, Curitiba, 8 (1-4): 31-73.

. 1996. Levantamento da fauna entomológica no Estado do Paraná. II. Ctenuchidae (Lepidoptera). Revista Brasil eira Zoologia, Curitiba, 13 (2): 435-461.

Marinoni, R.C.; R.R.C. D utra \& M.M. Casagrande. 1997. Levantamento da fauna entomológica no Estado do Paraná. III. Saturniidae (Lepidoptera). Revista Brasileira Zoologia, Curitiba, 14 (2): 473-495.

Marinoni, R.C.; R.R.C. Dutra \& O.H.H. Mielke. 1999. Levantamento da fauna entomológica no Estado do Paraná. IV. Sphingidae (Lepidoptera). Diversidade alfa e estrutura de comunidade. Revista Brasileira Zoologia, Curitiba, 16 (Supl. 2): 223-240.

Martin Piera, F. 1997. Apuntes sobre Biodiversidad y Conservación de Insectos: Dilemas, Ficciones y Soluciones? Sociedad Entomologica Aragonesa, Madrid, 20: 25-55.

$M_{A Y}$, R.M. 1988. How many species arethere on earth? Science, Washington, 241: 1441-1449.

Metrmam, J.C. 1999. Lepidoptera of Belize. 2. Catal og of emperor mothsand hawk moths (Lepidoptera: Saturniidade, Sphingidae). Tropical Lepidoptera, Gainesville, 10 (Suppl. 1): 
33-44.

Minshall, G.W.; Petersen, R.C \& N ımz, C.F. 1985. Speciesrichness in stream of different size from the some drainage basin. American Naturalist, Chicago, 125: 16- 38.

МотА, S.F. 1977. M eteorol ogia Agrícola. São Paulo, Biblioteca Rural Livraria Nobel, 3a ed., 376p.

Motta, C.S.; F.J. Aguiler-Peralta \& R. Andreazze. 1998. Aspectos da esfingofauna (Lepidoptera, Sphingidae), em área de terra-firme, no Estado do Amazonas, Brasil. ActaAmazônica, Manaus, 28 (1): 75-92.

PereIRA, J.M.M. 1994. Índicesfaunísticos eflutuação populacional de lepidópteros daninhos ao eucalipto na região de Montes Claros, MG. Anais da Sociedade Entomológica do Brasil, Londrina, 23 (2): 327-334.

Pereira, J.M.M.; J.C. Zanuncio; J.H. Schoereder; E.C. Nascimento. 1995. Agrupamento de oito povoamentos florestais em relação à fauna de lepidópteros daninhos ao eucal ipto, através de análise de agrupamento. Revista Brasileira de Entomologia, São Paulo, 39 (3): 647-652.

Silveira Neto, S.; Lara, F.M.; M. Fanzolin. 1977. Quociente e porcentagem de similaridade entre as comunidades de noctuídeos em Jaboticabal e Piracicaba, SP. Científica, São Paulo, 5 (3): 257- 261.

Silveira Neto, S.; R. Monteiro; R.C. Zucchi; R.C.B. Morais. 1995. Uso da análise faunística de insetosna avaliação do impacto ambiental. Scientia Agricola, Piracicaba, 52 (1): 9-15.

StiLING, P. 1999. Ecology. Theories and Applications. Apper Saddle River, New Jersey, Prentice Hall, $3^{\text {rd }}$ ed., XVII+638p.

Stradling, D.J.; C.J. Legg \& F.D. Bennett. 1983. Observations on the Sphingidae (Lepidoptera) of Trinidad. Bulletin of Entomological Research, Wallingford, 73: 201-232.

Tortowhater, C.W. \& J.R. Mather. 1955. The Water Balance. Climatology, Ceterton, 8 (1): 140.

Zimmerman, B.L. \& M.T. Rodrigues. 1990. Frogs, snakes, and lizards of the INPA-WWF Reserves near Manaus, Brasil, p. 426-454. In: A.H. Gentry (Ed.). Four Neotropical Rai nforests. New Haven, Yale University Press, 640p.

Xu, Chong-Yu. 1999. Operational testing of a water balance model for predicting climate change impacts. Agricultural and Forest M eteorology , Amsterdam, 98-99: 295-304.

Recebido em 09.I.2003; aceito em 09.VII.2004. 